\title{
LE SYSTÈME DE PENSÉE DE MAURICE HALBWACHS
}

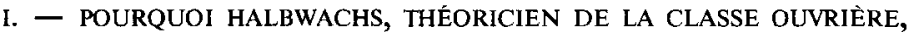 \\ NE POUVAIT ÊTRE À L'ORIGINE DE LA SOCIOLOGIE DU TRAVAIL \\ FRANÇAISE DE LA SECONDE MOITIÉ DU $\mathrm{XX}^{E}$ SIĖCLE
}

Un partage a été fait, dans l'œuvre de Maurice Halbwachs (1877-1945) qui s'étend sur toute la première moitié de ce siècle et qui est d'une diversité considérable, entre la part vivante et la part morte ou contestable ${ }^{1}$.

Empruntant le concept de morphologie sociale à son maitre Durkheim, Halbwachs est celui qui en a développé les implications dans une variété de travaux qui ambitionnent de renouveler la démographie et l'économie, et qui vont de l'étude des processus de la croissance urbaine à celle des conditions matérielles des institutions sociales. En particulier, on peut le considérer, sinon comme le premier ${ }^{2}$, du moins comme le plus éminent des introducteurs du point de vue sociologique dans la recherche urbaine qu'il juge illégitimement accaparée jusque-là par l'histoire et par l'économie classique. Dans une thèse qu'il a soutenue devant les juristes et les économistes de la faculté de droit, c'est à lui qu'on doit la première analyse de la spéculation foncière et immobilière ${ }^{3}$.

1. Sur l'œuvre publiée de M. Halnwachs, voir la bibliographie établie par Victor Karady à la fin de Classes sociales et morphologie, textes de Maurice HalBwaCHS, prés. de V. Karady, Paris, Minuit, 1972. Pour diverses appréciations de l'œuvre, se reporter en outre à : M. HALBWACHS, Esquisse d'une psychologie des classes sociales, avec une " Notice sur l'auteur ", de Georges Friedmann, Paris, Marcel Rivière, 1955; ID., Morphologie sociale, rééd. à Paris, Armand Colin, 1970, avec une "Présentation " d'Alain Girard; Michel Verret, " Halbwachs ou le deuxième âge du durkheimisme », Cahiers internationaux de sociologie, vol. LIII, 1972, p. 311-336.

2. Cf. Gilles MoNTIGNy, Les Études urbaines françaises en sociologie, géographie et statistique sociale, 1890-1920, thèse pour le doctorat de l'École des hautes études en sciences sociales, Paris, 1989.

3. Cf., entre autres contributions, "La politique foncière des municipalités », 1908, repris in V. KaRADY, op. cit. supra n. 1 ; Les Expropriations et le prix des terrains à Paris (18601900), thèse de doctorat en droit, Paris, Cornély, 1909; "Les plans d'extension et d'amé-

Revue de synthèse : IV S. Nº 2, avril-juin 1991. 
C'est, en outre, avec Halbwachs que la sociologie durkheimienne s'enrichit de l'étude des classes sociales, et même qu'elle devient l'étude de la société comme société de classes ${ }^{4}$. Au sein de la hiérarchie sociale, Halbwachs a examiné, avec un soin particulier et renouvelé, la condition de la classe ouvrière, à laquelle il a consacré sa thèse de doctorat d'État et plusieurs autres travaux, livres ou articles. Il est incontestablement le premier sociologue français à embrasser, sous le même regard, la sphère de la production et la sphère de la consommation, et surtout à théoriser l'articulation de la première à la seconde et, plus généralement, à montrer comment cette articulation joue différemment le long de la hiérarchie sociale tout entière. Lorsqu'il en vient à analyser les budgets de consommation ouvrière, à partir d'enquêtes empiriques réalisées en Allemagne, il élabore une méthode, qui a certes été perfectionnée depuis sur des points particuliers, mais qui fait aujourd'hui encore figure de modèle ${ }^{5}$.

Le thème de la mémoire collective apparaît dans l'œuvre plus tardivement. Il désigne, dans la pensée de l'auteur, un concept clé, qui continue d'être repris à l'heure actuelle chez des auteurs d'inspirations diverses ${ }^{6}$. Cette énumération est loin d'épuiser la richesse des thèmes abordés par Halbwachs. Surtout, elle ne rend pas compte de l'unité systématique qui les relie les uns aux autres, ni de la présence simultanée d'à peu près tous les thèmes à chaque moment du temps de l'œuvre. Par exemple, la mor-

nagement de Paris avant le $\mathrm{XIX}^{\mathrm{e}}$ siècle ", 1920, repris in V. Karady, op. cit. supra n. 1 ; « Le développement d'une grande ville : Chicago ", Annales d'histoire, 8, 1936, republié in L'École de Chicago. Naissance de l'écologie urbaine : textes, trad. et prés. par Yves GrafMEYer et Isaac Joseph, Paris, Aubier/Champ urbain, 1979.

4. Le cadre général de la théorie des classes est déjà fixé dans le premier essai que Halbwachs consacre, en 1905, au problème (cf. « Remarques sur la position du problème sociologique des classes ", Revue de métaphysique et de morale, 13, 1905, texte repris in V. KARADY, op. cit. supra n. 1). La théorie se développe dans La Classe ouvrière et les niveaux de vie. Recherches sur la hiérarchie des besoins dans les sociétés industrielles contemporaines, Paris, Alcan, 1913, rééd. Paris/Londres/New York, Gordon \& Breach, 1970. La clé définitive apparait dans Les Cadres sociaux de la mémoire, Paris, Alcan, 1925, réed. Paris, Presses universitaires de France, 1952. Pour la synthèse, voir Les Classes sociales, multigraphié, Paris, Centre de documentation universitaire, "Les cours de la Sorbonne ", 1937.

5. Cf., par ex., Christian Baudelot, Roger Establet et al., Qui travaille pour qui ?, Paris, Maspero, 1979, p. 51 : «C'est à M. Halbwachs que l'on doit les principes d'une analyse scientifique des budgets. Sa méthodologie et ses résultats ne sont en aucun cas dépassés. » Cf. également : Paul-Henry Chombart de LAuwe, La Vie quotidienne des familles ouvrières, Paris, Centre national de la recherche scientifique, 1956, passim. Cet ouvrage comporte de multiples évocations de Halbwachs, considéré comme un des grands prédécesseurs dans la voie des études portant sur le comportement de consommation de la classe ouvrière.

6. Cf. Les Cadres sociaux de la mémoire, op. cit. supra n. 4 ; La Topographie légendaire des Évangiles en Terre Sainte. Étude de mémoire collective, Paris, 1941; «Mémoire et société ", publication posthume, L'Année sociologique, $3^{\circ}$ série, vol. 1940-1948, texte republié sous le titre La Mémoire collective, Paris, Presses universitaires de France, 1950, $2^{e}$ éd., 1968. Pour une utilisation rigoureuse du concept de mémoire collective hors de son champ d'origine, cf. Gérard Nolriel, Les Ouvriers dans la société française, $x I X^{e}-X X^{e}$ siècle, Paris, Seuil, 1986. 
phologie sociale n'est pas indépendante de la réflexion sur les classes. Ainsi, l'étude inaugurale de la morphologie sociale sur les expropriations à Paris est en même temps une étude des besoins et du comportement de la bourgeoisie à l'égard du logement, dont la différence avec celui de la classe ouvrière est analysée jusque dans ses implications politiques. L'étude des cadres sociaux de la mémoire est simultanément une analyse des comportements de classe et du fonctionnement de la mémoire collective.

Voilà donc une pensée qui couvre une exceptionnelle diversité d'objets et qui se signale par un degré de cohésion tout aussi exceptionnel. Dans ces conditions, l'étonnant est qu'on ait pu la désarticuler pour n'en retenir que certains éléments : le thème de la mémoire collective, mais pas celui des classes; ou, dans l'analyse du comportement de la classe ouvrière, seulement la méthode d'analyse des budgets de consommation... Il y a plus : dans l'atmosphère de la sociologie française des années 1930-1940, raréfiée par les massacres de la guerre de 1914 qui n'épargnèrent pas la relève des jeunes sociologues, trois disciples de Durkheim occupent pratiquement tout l'espace : Mauss l'anthropologue, Simiand le spécialiste de la question du salaire, Halbwachs le sociologue au vaste registre. Or, lorsque, après l'interruption de la Seconde Guerre mondiale, redémarre la sociologie française du travail et des relations industrielles, elle le fait sans référence ni à Durkheim ni à Halbwachs, et va chercher ses modèles chez les Américains. Durkheim, théoricien de la division du travail, Halbwachs, théoricien de la classe ouvrière au travail et hors du travail ${ }^{7}$ !

On le sait, c'est Georges Friedmann qui est considéré unanimement comme le principal refondateur de la sociologie du travail en France. Dans les ouvrages successifs qu'il écrit entre les années trente et les années cinquante, Friedmann, qui fut d'abord proche du marxisme, s'en dégage rapidement et intervient de trois façons. Il disqualifie le point de vue durkheimien comme philosophique et dogmatique; il importe les résultats des enquêtes américaines; et il expose le fruit de ses propres observations et analyses, à partir d'une problématique qui s'interroge, pour l'essentiel, sur les effets sociaux du progrès technique ${ }^{8}$.

7. Cf., parmi beaucoup d'autres références possibles, Jean-René Treanton, «Sur les débuts de la sociologie du travail », Revue française de sociologie, oct-déc. 1986.

8. Dans La Crise du progrès. Esquisse d'une histoire des idées, 1895-1935, Paris, Gallimard, 1936, G. Friedmann évoque «la poigne solide de Durkheim [qui] maintenait dogmatiquement tout un groupe d'amis et de collaborateurs » (p. 33). Une formule qui prend place dans l'unique paragraphe de ce livre sur l'histoire des idées, consacré à « l'École sociologique française $\%$. Dès lors, on peut imaginer que la coïncidence est purement fortuite entre la date de 1895, choisie par Friedmann comme point de départ de son étude, et la publication des ouvrages fondateurs de la sociologie durkheimienne (Les Règles de la méthode 
Les raisons d'une telle rupture sont déjà un point d'histoire de la pensée qui mérite d'être éclairci. L'affaire prend tout son intérêt quand on s'aperçoit que Friedmann lui-même s'en est expliqué dans l'article nécrologique qu'il a consacré à Halbwachs en $1946^{\circ}$. Il conclut et résume la critique qui suit un éloge chaleureux de l'homme et de certains aspects de son œuvre, en jugeant que Halbwachs, en quelque sorte, est resté à michemin : ses travaux, écrit-il, constituent un remarquable pas en avant fait au-delà de l'orthodoxie durkheimienne, demeurée trop dogmatique et abstraite :

" Halbwachs se sépare de son maître et le dépasse lorsqu'il met en relief, dans un groupe social déterminé, à savoir la société industrielle contemporaine, les rapports de production qui conditionnent la mentalité des individus, leur sensibilité, leur conduite sociale, leurs besoins. Par là, en même temps, il se rapproche de la sociologie marxiste. "

D'une sociologie marxiste, faut-il le souligner, qui préexiste à celle de Durkheim! Friedmann crédite en quelque sorte Halbwachs d'avoir aidé à refouler Durkheim en le dépassant, mais reproche à sa pensée de n'être qu'une étape transitoire vers le retour à Marx...

Plus précisément, si Friedmann trouve féconde l'idée de rechercher le sens des comportements de consommation en les rapportant aux

sociologique, 1895; Le Suicide, 1897). Ses références, à l'époque, viennent d'ailleurs, et notamment de LÉNINE, dans L'Impérialisme, stade suprême du capitalisme, 1917, qui situe à cette époque l'ouverture d'une nouvelle étape du développement du capitalisme bancaire. $\mathrm{Cf}$. La Crise du progrès, op. cit., p. 257, n. 15.

On notera en outre que, lorsqu'il publie son ouvrage, Friedmann, qui a d'abord passé l'agrégation de philosophie (comme Halbwachs), achève la période de formation sociologique dont il a pu bénéficier entre 1931 et 1934 au Centre de documentation sociale de l'École normale supérieure, créé par Bouglé, disciple de Durkheim. Il a mis à profit tout ce temps pour faire un apprentissage sur machines-outils, enquêter dans les usines de divers pays et accomplir plusieurs séjours en Russie soviétique, après avoir appris le russe. Ses repères sont alors Marx, Lénine, et aussi Taylor, Ford...

Le point de vue de Friedmann sur la sociologie française n'a guère changé dans Où va le travail humain? Paris, Gallimard, 1950, p. 198-199 : "La sociologie française, née sous l'impulsion d'un génial bâtisseur de système, Auguste Comte, a conservé avec Durkheim, dont les mérites, par ailleurs sont immenses -, son caractère dogmatique et philosophique [...]. Les oeuvres de Lévy-Bruhl, Mauss et Halbwachs, déjà fort excentriques par rapport à l'orthodoxie du maitre de l'École sociologique française, beaucoup plus souples et plus riches de faits observés, n'en sont que plus méritoires. "

Sous la libérale direction de Friedmann et à son exemple, c'est vers l'Amérique que se tournent les jeunes sociologues de l'époque à la recherche d'une tradition sociologique. Alain Touraine se définit de façon polémique, en particulier, par rapport à Parsons. Derrière Parsons : Max Weber. De même pour Crozier : son analyse de la bureaucratie prend pour point de départ le modèle de Max Weber, corrigé et enrichi par le mouvement des « human relations ", lui-même héritier d'influences diverses, anthropologiques, psychanalytiques, etc.

9. Paru dans la revue Europe, de janvier 1946, et reproduit sous le titre "Notice sur l'auteur ", en tête de l'Esquisse d'une psychologie des classes sociales, Paris, Marcel Rivière, 1955 , citations extraites des pages 17 et 15 . 
comportements de production, il ne s'en prend cependant ni plus ni moins qu'à l'essentiel de la pensée de Halbwachs : il conteste que celui-ci interprète correctement le sens de la consommation, aussi bien que celui du travail des ouvriers.

Halbwachs, en effet, dresse la hiérarchie des besoins qui caractérise en propre la classe ouvrière, en constate la relative plasticité, et conclut que cette plasticité exprime une liberté de choix subjective qui pourrait être mieux employée - par exemple, en consacrant moins d'argent à la nourriture et plus à l'amélioration du logement. On sent que Friedmann, fils de grands bourgeois qui a travaillé de ses mains aux côtés des ouvriers, est choqué de l'insensibilité de Halbwachs à la misère ouvrière : les variations observables dans le profil des dépenses ouvrières seraient mieux interprétées, dit-il en substance, si on les voyait comme la traduction des variations objectives de la pénurie à laquelle s'adaptent, forcés, les besoins de la classe ouvrière.

Même critique, elliptique et retenue, de la signification sociale que Halbwachs attribue au travail ouvrier :

"Cette étude scientifique de la hiérarchie des besoins est encore soustendue, chez Halbwachs, par une curieuse théorie [...] de la place occupée par les ouvriers dans la société capitaliste contemporaine. Celle-ci prépose à la fonction de $[\ldots]$ produire, un ensemble défini de leurs membres - la classe ouvrière - qui, pour s'en acquitter, "sont contraints de rester en contact avec les choses, de s'isoler en face d'elles et de se détacher du reste de la collectivité humaine $" »{ }^{10}$.

Friedmann n'en dit pas plus et laisse à d'autres le soin de fouiller la discussion de cette curieuse théorie de l'ouvrier absorbé par la matière et séparé de la société. Sans doute pensait-il mieux faire en développant sa propre théorie de la civilisation du travail - du travail comme facteur de civilisation. Mais il se trouve que la discussion que Friedmann appelait (sans réelle conviction, semble-t-il) de ses vœux, Michel Verret l'a faite en 1972 dans un article qui fait magistralement ressortir les mérites de l'œuvre de Halbwachs ${ }^{11}$.

Ces mérites n'empêchent pas, selon Verret, que l'analyse de Halbwachs ne reste " paradoxalement marquée, dans sa dénonciation des effets de domination de classe, par les effets d'ethnocentrisme que cette domination induit dans l'idéologie de la classe dominante ". Car la théorie de l'aliénation ouvrière, que Verret lit dans Halbwachs, est prise dans une

10. Ici Friedmann cite un passage de "Matière et société ", article de Halbwachs paru dans la Revue philosophique en 1920 et reproduit in V. KARADY, op. cit. supra n. 1.

11. Cf. art. cit. supra n. 1, citations extraites des pages 329 à 335 . 
"présomption de désocialisation". Entendons par là que, en fin de compte, la classe ouvrière aliénée n'est pour Halbwachs que le «pur négatif des classes dominantes ", le degré zéro de la socialité ", et que l'ouvrier n'apparait que comme le "primitif des sociétés occidentales". Verret ajoute, dans une formule qui condense lumineusement le raisonnement de Halbwachs :

«Si l'on définit la vie collective par l'intensité et la variabilité de la relation interhumaine, la relation interhumaine par l'exclusion de la relation aux choses et la classe ouvrière par la relation exclusive aux choses, la classe ouvrière se trouve évidemment exclue par définition de la socialité et de l'humanité. "

D'où cette conséquence que " la sociologie du travail trouverait $[. .$. paradoxalement sa limite à la porte de l'usine [...]».

L'analyse de Verret aide à comprendre pourquoi l'œuvre de Halbwachs consacrée à la classe ouvrière au travail et hors du travail n'a été d'aucune ressource dans le démarrage véritable d'une sociologie du travail qui réintégrait les usines dans la société. Elle ne s'en tient pas là, et recherche la cause d'un " aveuglement sociologique " qui " étonne chez un théoricien aussi attentif au siècle». Elle la trouve dans "l'allégeance idéaliste" de Halbwachs à la définition durkheimienne des faits sociaux, qui fait d'eux de simples représentations collectives. En donnant une part trop exclusive aux idées, Halbwachs aurait indûment exclu de la sociologie la prise en considération des conditions matérielles du travail. Non que tout matérialisme soit absent de ses préoccupations : la morphologie sociale, à laquelle il a accordé une si grande attention, n'est rien d'autre que l'étude du " corps » de la société et des déterminations matérielles des représentations collectives. Mais, selon Verret, le matérialisme de Halbwachs est régressif par rapport à celui de Marx, car il est toujours second par rapport aux représentations, jamais déterminant. L'" ethnocentrisme de classe ", pris dans une " théorie idéaliste de l'objet social ", limite irréversiblement la sociologie de Halbwachs, marquée par «la prohibition de Marx ".

Remarquable convergence: Friedmann reste interloqué devant la " curieuse théorie " de Halbwachs, cependant que Verret s'" étonne " de l'aveuglement d'un sociologue d'aussi large envergure. Le premier le voit arrêté à mi-chemin de son avancée de Durkheim à Marx; le second lui fait crédit d'être d'emblée parvenu à une théorie de l'aliénation qui l'aurait rangé au même niveau que Marx s'il n'était resté empêtré dans les présupposés de l'idéalisme durkheimien. Halbwachs, entre Durkheim et Marx, ou entre Marx et Durkheim? 
Notre propos est de reprendre à notre tour la " curieuse théorie " qui nous a intrigué, autant que nos prédécesseurs. Pour ce faire, il faut d'abord, une fois pour toutes, cesser de référer Halbwachs à Marx et, surtout, de le lire à travers les lunettes de Marx. Alors, on découvre que Halbwachs n'était pas, dans un sens ou dans l'autre, à mi-chemin de Durkheim, qu'il connaissait, et de Marx, que pendant longtemps, il n'a connu que de loin et indirectement ${ }^{12}$; mais ailleurs, analysant le taylorisme avec les lunettes des aristocrates de l'Ancien Régime : une combinaison qui ne prédispose pas à comprendre positivement le sens de la civilisation industrielle ou du capitalisme.

En vérité, c'est seulement en 1925, dans Les Cadres sociaux de la mémoire, qu'apparaît cette référence, étonnante et inattendue, au point de vue aristocratique. Et c'est vraiment dans le cours sur Les Classes sociales de 1937 que Halbwachs en développe le plus nettement les tenants et les aboutissants.

Le dualisme qu'implique tout aristocratisme se surajoute de façon quasi naturelle à un autre dualisme, qui était présent dans l'œuvre de Halbwachs depuis ses débuts et qu'on trouve dès 1905 dans un article intitulé Remarques sur la position du problème sociologique des classes ${ }^{13}$. Ce dualisme-là est d'inspiration philosophique : il oppose l'esprit et la matière. Mais il a de multiples connotations et recouvre plusieurs oppositions : celle, durkheimienne, du fait social qui est, en son essence, du psychologique collectif - et des faits matériels non sociaux; celle, bergsonienne, de l'élan vital, qui est création spirituelle - et de ses retombées ou solidifications... L'opposition des dépositaires de la socialité par excellence (les aristocrates) et des autres se surimpose sans difficulté aux précédentes. Malgré ses sources composites, ce système de pensée est doté d'une rigidité élevée. Forcément! Le dualisme, par définition, ne porte pas à la nuance : comme dans toute logique binaire, le courant passe ou ne passe pas; ou bien on est dedans, ou bien on est dehors... Calant son alidade sur un astre qui avait depuis longtemps disparu sous l'horizon, entrant à reculons dans la société industrielle, et choisissant de démontrer selon la règle du tout ou rien, Halbwachs sociologue courait au désastre assuré.

12. Halbwachs, qui pratiquait l'allemand pour avoir fait en Allemagne un long sejour d'étude, comme beaucoup d'intellectuels de divers pays, à la fin du XIX siècle et jusqu'à l'aube de l'époque nazie, est demeuré longtemps avant de se familiariser directement avec l'œuvre de Marx. Dans sa thèse sur La Classe ouvrière et les niveaux de vie, op. cit. supra n. 4, il évoque, à plusieurs reprises, et réfute des idées qu'il attribue à Marx et qui proviennent du Manifeste communiste et de la longue préface que Charles Andler lui a ajoutée. C'est là, semble-t-il, toute sa provende.

13. Paru dans la Revue de métaphysique et de morale, 13, 1905, p. 890-905 ; article reproduit in V. KARADY, op. cit. supra n. 1. 
II. - " LA CLASSE OUVRIËRE RESSEMBLE BIEN PLUS, CROYONS-NOUS, À UNE MASSE MECANIQUE ET INERTE QU'À UN ORGANISME VIVANT ET SOUPLE ${ }^{14}$

Mais d'abord il convient de montrer en quoi le fait de chausser les lunettes de Marx fait voir dans l'œuvre de Halbwachs des choses qui n'y sont pas et, encore plus, empêche de voir celles qui y sont. La « théorie de l'aliénation" ouvrière dont Verret crédite Halbwachs n'existe pas. D'abord, "aliénation" est un mot qu'on ne trouve à aucun endroit de l'œuvre de Halbwachs. Ni d'autres expressions, comme « domination de classe " que Verret utilise pour résumer les analyses que donne Halbwachs de la condition ouvrière au travail.

"La séparation de l'ouvrier à l'égard de ses moyens de production, écrit Verret, et l'obligation où elle le réduit de vendre l'usage de sa force de travail à ceux qui les possèdent, le dépossède aussi à leur profit de toutes les fonctions de conception, de direction et d'organisation du travail [...]. Deux fois dépossédé des fonctions intellectuelles et directives du travail et des rapports interpersonnels qui s'y nouent, l'ouvrier, ainsi livré aux choses, devient luimême chose. "

Si ce n'est pas Marx qui écrit ces lignes, c'est sa pensée, à coup sûr, que rapporte Verret. En aucun cas, ce ne peut être celle de Halbwachs, pour qui, certes, l'ouvrier prend conscience "d'être surtout un instrument matériel et mécanique engagé dans la nature brute ", mais est avant tout, non pas la victime d'une aliénation dépossédante, mais l'objet d'une "délégation", ou encore, d'une "préposition" au travail de la matière ${ }^{15}$. La différence entre les deux registres est évidente : l'aliénation

14. La Classe ouvrière et les niveaux de vie, op. cit. supra n. 4, p. XVII.

15. Cf. ibid., p. 421 et 75 : «Comme des outils qui, dans leur poignée portent encore la marque humaine, mais qui sont essentiellement matière dans les parties par lesquelles ils agissent, les ouvriers doivent en quelque sorte se solidifier, se durcir, substituer à leurs contours naturels et humains des lignes plus angulaires, sur toute la façade de leur vie qui regarde les choses inertes, c'est-à-dire sur toute leur activité. Un philosophe contemporain a défini l'intelligence "la faculté de fabriquer des outils artificiels, en particulier, des outils à faire des outils". Nous dirions volontiers que la société, en constituant hors d'elle, ou plutôt en reportant hors d'elle toute une classe d'hommes délégués au travail matériel, a su fabriquer des outils à manier des outils. »

Texte stupéfiant, où se donne libre cours une mimétologie onirique (l'ouvrier, au contact des choses et des outils, se métamorphose en chose-outil), et où se développe une obsession phobique du contact avec la matière (il faut « reporter " ce genre de chose hors de la société. On notera que « report", ou " délégation ", n'est pas « aliénation ").

Toujours est-il que l'ouvrier n'est que l'outil rudimentaire d'un outil perfectionné. $\dot{A}$ un autre endroit de l'ouvrage, p. 118-119, Halbwachs évoque "l'obligation " que la société, pour agir sur la matière, doit imposer " aux groupes dont elle se sert ", de s'absorber 
implique un arrachement violent de l'individu à son être par un pouvoir illégitime, tandis que la délégation ou la préposition est de l'ordre de la fonctionnalité technique. Elle va tellement de soi que personne ne la met en question, ni hors de la classe ouvrière, ni même dans la classe ouvrière, qui se définit, selon Halbwachs, par la reconnaissance consciente de la hiérarchie de l'ordre social et par la place qu'elle occupe au plus bas degré de cette hiérarchie ${ }^{16}$.

Car une classe sociale, Halbwachs le pose comme axiome essentiel, n'existe pas sans conscience de classe, c'est-à-dire sans percevoir et accepter le rang qui lui est assigné dans la hiérarchie de la société, univoquement reconnue par toutes les classes qui la composent. Avec une indéfectible et tranquille obstination et d'un bout à l'autre de son œuvre, Halbwachs accumule les démonstrations sans nuance qui, toutes, concluent à l'unité de la classe ouvrière dans son isolement, dans l'acceptation de sa totale infériorité et, par conséquent, dans la ratification du jugement d'exclusion porté sur elle par le reste de la société.

Commençons par La Classe ouvrière et les niveaux de vie. Le chapitre de la première partie qui est consacré à l'analyse des conditions techniques et juridiques du travail ouvrier a pour objet de rechercher si les métiers, les industries, les modes de rémunération peuvent servir de support à la conscience ouvrière. Or il est clair que les métiers offrent une grande diversité, de même que les industries, et aussi, dans une moindre mesure, les modes de rémunération.

\footnotetext{
complètement dans la matière au point que « tout ce qui est humain, et surtout social leur devienne, pendant tout ce temps, étranger $»$. Le lecteur aura saisi le clin d'œil de l'humaniste, et compris que le célèbre vers de Pline (" Je suis homme, et rien de ce qui est humain ne m'est étranger "), ne vaut pas pour les travailleurs au travail. Hors travail, fort peu de ce qui est humain ne leur est pas complètement étranger, puisque le logement ouvrier est plus « animal " que " social ", et que la sexualité ouvrière est plus sauvage que civilisée, etc.

Halbwachs rejette la classe ouvrière hors de la société, c'est-à-dire hors de l'humanité. En commentaire de la formule sur l'absorption de l'ouvrier dans la matière, il cite, ibid., p. 119, n. 1, un texte de l'aristocrate grec Xénophon qui lui paraît convenir à l'opposition moderne du travail industriel et de la vie sociale. Le voici : « Les arts appelés mécaniques sont décriés. C'est avec raison que l'opinion publique en fait peu de cas. Ils ruinent le corps des travailleurs et de ceux qui les surveillent, contraints qu'ils sont à demeurer assis, à vivre dans l'ombre, parfois mème à séjourner près du feu. Or quand les corps s'efféminent, les âmes s'amollissent. De plus rien ne s'oppose davantage à l'accomplissement de nos devoirs envers nos amis et l'État. Aussi les travailleurs manuels passent pour de mauvais amis et de lâches défenseurs de la patrie " (XÉNOPHON, Économique, IV). Certes la guerre de 1914 n'avait pas eu lieu; mais elle n'a pas empêché Halbwachs de considérer que les ouvriers étaient tout autant exclus du lien social et des vertus qui l'accompagnent.

16. Cf. art. cit. supra n. 10, p. 62-63 : les ouvriers «... se sentent isolés, exclus, dépréciés... ». " Comme on ne s'expliquerait point [...] que la société se fermât à tant d'hommes, s'ils étaient qualifiés pour s'y introduire, et capables d'accroître le nombre et l'intensité des relations qui unissent ses membres, il faut conclure qu'ils ne possèdent ni ces qualités ni ce pouvoir. "
} 
Mais il n'est pas question, pour Halbwachs de laisser " s'éparpiller " l'unité de la conscience ouvrière en des fractions qu'aucun principe ne permettrait plus de dominer, et qui ne correspondraient plus à la représentation que la bourgeoisie se fait de cette classe comme d'une masse monolithique. Aussi tranche-t-il, au terme de raisonnements abstraits : rien ne peut l'emporter sur le grand dénominateur commun à tous ces éléments, qui est, non pas, bien sûr, l'aliénation ou quoi que ce soit de ce type, mais l'isolement de chacun des individus en face de la matière. Isolement qui est condition d'une conscience de classe négative ou privative, puisqu'il entraine l'abrutissement de la conscience ${ }^{17}$.

Se sentant tenu de répondre aux objections portées contre une thèse qui définit la classe ouvrière comme «l'ensemble des hommes qui, pour s'acquitter de leur travail, doivent se tourner vers la matière et sortir de la société ", Halbwachs va plus loin encore ${ }^{18}$.

On pourrait objecter, reconnait-il d'abord, que la matière traitée par l'homme dans le machinisme industriel est si élaborée et les machines si manifestement constituées d'intelligence humaine, que dans un produit, une machine ou un morceau de matière, la société ne retrouve " presque rien qui ne porte sa marque ". Si l'on ajoute que rien du travail ne se fait en dehors d'une organisation rationnelle de la coopération des hommes et de la division des tâches, il faudrait plutôt conclure que, loin d'être hors de la société dans l'usine, l'ouvrier y est dans « un milieu hypersocial au contraire », et que "l'activité ouvrière serait moins inhumaine que surhumaine " (p. 65). Une objection qui est un magnifique morceau de bravoure! Halbwachs va s'employer à la démolir avec une surprenante opiniâtreté.

Le leitmotiv du raisonnement est dans l'idée de la " nécessité de maintenir une ligne très nette de démarcation entre les ouvriers, quels que soient leur rendement et leur qualification » et les autres composantes de la société, de marquer "l'infériorité sociale » de la situation d'ouvrier, de montrer "le fossé " que "la société fait passer " entre le "travail" de l'ouvrier et l'« activité » de l'ingénieur, etc.

D'abord, la machine, faite de matière humanisée, ne rassemble pas les ouvriers, mais les sépare et les exclut. Le résidu de matière brute qui a résisté aux prises de la mécanisation, c'est ce qui suffit à occuper l'ouvrier, appendice marginal d'une machine dont il ignore le fonctionnement et qui est faite de l'incorporation d'une intelligence humaine qui n'est pas celle de la classe ouvrière.

17. Cf. La Classe ouvrière, op. cit. supra n. 4, p. 119 : «... l'ouvrier, dans l'état d'isolement où il se trouve ainsi d'habitude (...) peut vaguement penser et sentir... "

18. Cf. art. cit. supra n. 10. 
Avec l'organisation scientifique du travail proposée par Taylor, un pas de plus est accompli dans la "désocialisation » de l'ouvrier, qui paraissait pourtant impossible à pousser plus loin. Le premier principe de la méthode taylorienne est de décomposer en éléments simples le travail de l'ouvrier, qui n'est déjà qu'un travail résiduel, de telle sorte que le peu qui subsistait d'initiative dans l'exécution soit transféré hors de la conscience de l'ouvrier et reporté chez le chronométreur. Selon le second principe, les forces physiques de l'ouvrier seront elles aussi décomposées en leurs éléments, c'est-à-dire en aptitudes particulières, de sorte qu'on puisse ensuite sélectionner les ouvriers selon ces aptitudes, afin de les adapter sans marge aux travaux élémentaires. Halbwachs commente :

« De même qu'on a décomposé les résistances de la matière pour en mieux venir à bout, de même il faut décomposer les résistances physiques des hommes, pour exercer sur chaque résistance la force qui lui correspond le mieux : c'est un deuxième mode de division du travail qui ne s'impose pas moins que le premier" (p. 71).

Le système de Taylor, en individualisant les tâches, en interdisant la coopération, rend plus complète que jamais la "rupture" de l'ouvrier avec ses compagnons et avec la société. Il fait apparaître, en somme, que la thèse de Halbwachs, loin d'être excessive, était en deçà de la réalité.

Mais l'organisation scientifique du travail laisse de côté les ouvriers de métier qui paraissent échapper au sort commun de la désocialisation, grâce à la capacité qu'ils ont conservée de combiner jusqu'à la virtuosité une multitude de qualités complexes, et grâce à la pratique d'une autodidaxie qui les hisse, semble-t-il, au niveau des connaissances techniques de l'ingénieur et du savant. Les ouvriers, exclus de la société par une porte, vont-ils pouvoir y rentrer par une autre?

$\mathrm{Si}$ nous admettons de considérer, dit Halbwachs, que la "pensée ouvrière" peut aller jusqu'à la compréhension de la théorie, nous devrons admettre qu'elle s'engage aussi dans " une de ces avenues qui conduisent au cour même de la société", et nous devrons par conséquent faire rentrer au moins une catégorie d'ouvriers "vers la société dont nous prétendions l'exclure" (p. 84 sq.). Halbwachs, inflexible, ne fera cependant pas d'exception: " les bons ouvriers en restent retranchés comme les autres. » II tient au principe de l'unité de la classe ouvrière. Unité externe et interne. Externe, comme classe exclue en bloc. Interne, comme classe dont les membres sont unis par une " vague " solidarité. L'essai sur Mémoire et société se termine par cette formule où triomphe l'intransigeance démontrante de Halbwachs, mais qui plonge le lecteur d'aujourd'hui dans la plus extrême perplexité : «[...] l'ouvrier aura beau se pencher attentivement sur son métier : il n'y aper- 
cevra aucune facette qui lui renvoie l'image d'une activité sociale, c'est-àdire où la société se reconnaisse" (p. 94).

Mais pourquoi la société a-t-elle décidé, et Halbwachs avec elle, de condamner à l'asocialité perpétuelle la classe ouvrière et le travail de la matière ? C'est en répondant à cette question qu'on touchera au cour du système de pensée de Halbwachs et à ses difficultés.

\section{III. - LE SYSTÈmE DE PENSÉE DE halbWACHS : UN DUALISME ARISTOCRATIQUE DE LA MATIĖRE ET DE L'ESPRIT}

L'étrangeté du point de vue adopté par Halbwachs tient à la conception limitativement aristocratique que ce sociologue du $\mathrm{xx}^{\mathrm{e}}$ siècle se fait de l'essence du fait social, dont pourtant il emprunte la définition à Durkheim.

Dans un premier temps, Halbwachs reprend complètement l'héritage durkheimien. Il ne manque pas de souligner que le précepte qui commande de considérer les faits sociaux comme des choses est de simple méthode car, ce faisant,

« Durkheim n'entendait pas les assimiler aux faits de la physique, et les vider [...] de leur contenu spirituel. Mais il se défiait de la psychologie individuelle, fondée sur l'observation intérieure ${ }^{19}$.

Il n'a pu constituer la sociologie qu'en l'arrachant à la psychologie introspective. Mais les faits sociaux appartiennent à l'ordre de la conscience, de la représentation psychique, du mental, de l'esprit ou de l'âme, tous termes qui se substituent les uns aux autres sous la plume de Halbwachs. Leur spécificité, dans la sphère des faits psychiques, est d'être collectifs, c'est-à-dire " extérieurs" aux consciences individuelles.

C'est dans ce contexte épistémologique que, jusque vers 1925, Halbwachs analyse les faits sociaux et, en particulier, qu'il conçoit sa théorie de l'exclusion de la classe ouvrière hors du foyer central de la socialité. Sa thèse sur La Classe ouvrière et les niveaux de vie est contemporaine du livre de Durkheim sur Les Formes élémentaires de la vie religieuse (1912). Le second voyait dans les religions primitives les formes élémentaires des religions développées; le premier voit dans l'ouvrier, pour reprendre la formule de Verret, le primitif des sociétés industrielles.

19. Cf. l'introduction de "La doctrine d'Émile Durkheim ", Revue philosophique, 85, mai-juin 1918. 
Puis, un peu plus tard, dans Les Cadres sociaux de la mémoire, Halbwachs imprime à la définition durkheimienne du fait social une inflexion résolument passéiste, qui fait de lui un homme des temps pré-industriels en plein $\mathrm{xx}^{\mathrm{e}}$ siècle. C'est trop peu de dire qu'il était enfermé dans le point de vue ethnocentrique des classes dominantes. C'est l'ancienne noblesse disparue qu'il considérait comme le terme de référence idéal. Elle représente pour lui l'illustration par excellence de l'autonomie spécifique du fait social, indépendant de toute nécessité extérieure, spiritualité pure échappant aux pesanteurs de la matière. Dans le cours de la Sorbonne sur Les Classes sociales, la noblesse occupe un chapitre décisif, malgré sa faible importance numérique (quelques milliers de personnes). C'est que " le genre de pensée et de vie étranger aux nécessités matérielles " qui est caractéristique de la noblesse d'autrefois, constitue le "couronnement " de l'organisation sociale. Les gens titrés, nous dit Halbwachs,

«croient réellement que leur groupe est la partie la plus précieuse, la plus irremplaçable, en même temps que la plus active et bienfaisante du corps social, qu'il est, en un sens, la raison d'être de la société. Il faut analyser cette croyance qui ne se ramène pas à un simple entraînement de vanité collective, et qui est fondée sur une appréciation assez exacte de la nature et du rôle d'une classe noble ${ }^{20}$.

En quoi consistent la nature et la fonction de la noblesse ? À ne baigner que dans les relations sociales, vingt-quatre heures sur vingt-quatre... La noblesse est un groupe dans lequel tout le monde se connaît (ou peut se connaître) et ne passe son temps qu'à connaitre tout le monde intuitu personae, en étant capable de remonter la chaîne des liens qui unit toutes les personnes à travers le temps. En d'autres termes, la noblesse est un groupe qui s'autoconnaît comme groupe et qui n'a d'autre finalité que de se connaître lui-même. Mais, objectera-t-on, si ce sont des personnes, qui se connaissent les unes les autres, comment prétendre que le groupe se connait comme groupe ? et comment, tout simplement, la sociologie estelle encore possible? C'est que, pour Halbwachs, la " personne " n'est rien d'autre que ce qui est social dans l'individu. Elle n'existe, dironsnous, qu'en représentation pour elle-même et pour les autres ${ }^{21}$. D'où l'importance de la prouesse et de l'exploit, qui attestent que les aptitudes

20. Cf. Les Cadres sociaux de la mémoire, op. cit. supra n. 4, chap. VII, p. 309.

21. Cf. ibid., p. 312-313: « ... chaque noble ou chaque famille noble est plongée si profondément dans l'ensemble des autres familles de même classe, qu'elle les connait (ou est censée les connaître) toutes, et que, d'autre part, toutes la connaissent et connaissent ses origines, sa place et ses ramifications dans leur groupe. Deux nobles qui se rencontrent sans s'être jamais vus doivent être en mesure, après un échange de quelques propos, de se reconnaître comme deux membres d'une famille étendue [...]. Ceci suppose que se perpétue dans la classe noble, à travers les générations, tout un ensemble bien lié de traditions et de 
mises en l'individu par la nature humaine ont bien été cultivées pour leur propre illustration et non dans la subordination à une fin extérieure. Le monde social qui définit l'humanité de l'homme est un monde de finalité sans fin, où toutes les qualités humaines ne sont valorisées que pour ellemêmes, dans un état de grâce et d'apesanteur que ne trouble aucune contrainte extérieure. Monde sans travail, monde ludique, monde esthétique !

Tel est le plan où se meuvent et s'évaluent les personnes, en un monde de relations sociales où rien ne rappelle la production et la matière. Un degré en dessous, se situe le plan des "fonctions". La noblesse, qui consacre tout le loisir de son temps à la cérémonie, à la parade et à la représentation, n'a jamais exercé de fonction, un genre d'activité qui

" limite et dénature la vie sociale et représente comme une force centrifuge qui tend à écarter les hommes du coeur de la société. Pour exercer l'une d'elles, il faut que les hommes, temporairement au moins, s'abstiennent des autres $"{ }^{22}$.

Il $\mathrm{y}$ a fonction dès qu'on ne traite plus les individus et les familles comme des cas particuliers de personnes insérées dans le souple et vivant tissu de la mémoire collective, mais comme des généralités. Alors on cesse de tenir compte seulement " de l'honneur, du prestige, des titres, c'est-à-dire de notions purement sociales, où n'entre aucun élément de nature physique qui se prête à la mesure, au calcul, ou à une définition abstraite ${ }^{23}$. En traitant les êtres humains dans l'anonymat des règles générales, on commence à les traiter "comme des choses" et "mécaniquement ". Ne les saisir que comme des métaphores de choses, c'est commencer à sortir de la pure socialité et, tout simplement, de la société.

Un degré plus bas, la fonction devient simple technique, c'est-à-dire application complètement "mécanique" de la règle, sans liberté d'appréciation ${ }^{24}$. Tout en bas enfin, n'existe que le contact, non plus métaphorisé mais direct, avec la matière elle-même, c'est-à-dire la subordination totale des aptitudes déposées en chacun par la nature humaine à une finalité complètement extérieure : la fabrication d'un produit maté-

souvenirs. Comme rien de semblable ne se rencontrait dans les autres groupes, il faut dire que la classe noble a été longtemps le support de la mémoire collective. »

22. Ibid., p. 310 .

23. Ibid, p. 312.

24. La démultiplication de la fonction et de la technique n'est pas dans Les Cadres sociaux... Elle apparaît plus tard, lorsque Halbwachs se décide à autonomiser les classes moyennes au sein de la bourgeoisie. L'attribut principal des classes moyennes, c'est l'application pure et simple de " techniques ». Cf. " Les caractéristiques des classes moyennes », in Inventaires III, Paris, Alcan, 1939, texte reproduit in V. KARADY, op. cit. supra n. 1. 
riel, dans le cadre d'un métier ou d'un travail parcellaire qui n'ont pas leur fin en soi.

Une image traduit la différence entre la finalité sans fin de l'activité de la personne et l'activité, subordonnée, du travail matériel : la comparaison du couvreur et de l'alpiniste. Tous deux développent la même aptitude à vaincre audacieusement le vertige; mais, le premier, pour une fin qui lui est dictée de l'extérieur et pour aboutir à la production d'un objet matériel, extra-social par définition; le second, surtout pour rien, et pour que soit reconnue et appréciée seulement son audace. Car l'axiome ethnocentrique, qui commande en fait l'analyse de Halbwachs, consiste à poser que la société valorise comme sociales les aptitudes qui sont mises en œuvre dans l'élément de la gratuité aristocratique, et déprécie comme indignes de figurer au tableau d'honneur de la société les aptitudes qui s'exercent à des fins de production ${ }^{25}$.

La hiérarchie des activités, établie selon leur degré de subordination à des fins de plus en plus socialement dépréciées, permet de dresser le tableau et la hiérarchie sociale. Au sommet, la noblesse hors d'atteinte de la matière et s'adonnant totalement au loisir de la socialité. Admiratif, Halbwachs note l'aisance aérienne avec laquelle un noble se meut à travers tous les milieux : en chaque être, aristocrate ou manant, il ne voit que la personne et n'est en rapport qu'avec elle ${ }^{26}$. Un degré en dessous, la bourgeoisie se définit par l'exercice des «fonctions »; un degré en dessous encore, les classes moyennes sont telles, parce que la société ne peut plus leur confier que l'application des techniques. En bas, au contact de la matière organique et du sol sont les paysans et, au contact de la matière physique, les ouvriers.

La bourgeoisie moderne, à vrai dire, ne se réduit pas à la pratique des fonctions. Elle est «l'héritière » de la noblesse. D'abord, dans tout le temps qu'elle ne consacre pas aux fonctions, elle vit intensément au sein de groupes familiaux et mondains où elle n'a d'autre préoccupation que d'enrichir sa vie spirituelle.

«Ces groupes : famille, monde, etc., ne jouent-ils pas par rapport aux professions, le même rôle dont s'acquittait autrefois la classe noble, par rapport

25. Ce que valorise Halbwachs est exactement ce qui excite les sarcasmes de Thorstein VEBLEN in Théorie de la classe de loisir, 1899, trad. fr. Paris, Gallimard 1970, et inversement. Le plus étrange est de constater que Halbwachs cite souvent, comme allant dans le sens de sa propre thèse, les conduites de consommation ostentatoire et de gaspillage que Veblen attribue à la bourgeoisie et dans laquelle il voit une perversion de la socialité. Pour Veblen, le goût du travail utile et bien fait (« workmanship »), qui caractérise l'homme de travail, est au principe de la véritable socialité. Mais Veblen ne rejetait pas pour autant les bourgeois spéculateurs et gaspilleurs hors de l'humanité sociale.

26. Cours sur Les Classes sociales, op. cit. supra n. 4, p. 96 . Le manant a droit à plus d'égards, de la part de l'aristocrate, que l'ouvrier-masse, de la part d'Halbwachs. 
aux fonctionnaires et aux fonctions? Et puisque la noblesse était le support des traditions, et que la mémoire collective vivait en elle, n'est-ce pas dans la vie sociale extra-professionnelle, telle qu'elle est organisée aujourd'hui, que la société conserve et élabore ses souvenirs? $"{ }^{27}$.

Mais il y a plus. Pour Halbwachs, la bourgeoisie actuelle est capable d'endiguer l'intrusion de la matière (métaphorisée ou réelle) et même de prendre l'offensive en introduisant le social au sein même des «fonctions " professionnelles. Non seulement elle s'arrache aux déterminations objectives et matérielles de la profession en développant sa conscience de classe sociale dans la sphère extra-professionnelle du loisir; mais en outre, faisant mieux encore que l'aristocratie, elle se comporte dans l'exercice de ses fonctions comme si elles étaient purement sociales, et dans l'exercice des relations professionnelles comme si elles étaient purement extra-professionnelles ${ }^{28}$. La bourgeoisie vue par Halbwachs traite le négoce (neg-otium, étymologiquement : la privation du loisir) comme un loisir (otium)! En passant du monde extra-professionnel et familial au monde professionnel, elle ne quitte jamais la société et ne vit qu'immergée dans les relations sociales ${ }^{29}$.

Si l'on se représente la société comme une pyramide hiérarchique de strates distinctes, on voit que le dualisme aristocratique de la matière et de l'esprit affecte (ou infecte?) ces strates de façon inégale. Plus on monte dans la hiérarchie, plus la part et le poids de la matière s'amenuisent dans

27. Cf. Les Cadres sociaux, op. cit. supra n. 4, chap. vil, p. 328.

28. Dans "Les caractéristiques des classes moyennes", in op. cit. supra n. 24, p. 108, Halbwachs prend souvent l'exemple du magistrat, qui peut avoir à traiter des cas simples, où l'application "mécanique " de la loi pourrait facilement être sous-traitée par un greffier des classes moyennes. « Mais il se présente de temps en temps des cas où cette technique ne suffirait pas, où il faut envisager les dispositions psychologiques, morales, des sujets que l'on a à juger [...]; on doit songer à la classe à laquelle appartient l'accusé, à son rang social, à sa famille, à son origine, à toute espèce de mobiles et de motifs pour l'examen desquels il faut une certaine connaissance de la vie des hommes, et surtout du monde et des personnes, connaissance qui ne se développe que dans les milieux de la bourgeoisie. " Cf. Les Cadres sociaux, op. cit. supra n. 4, chap. vi, p. 331 sq. : “ Un juge peut avoir à juger [...] des personnes qu'il est exposé à rencontrer dans le monde, ou qui [...] évoquent en lui l'image de parents ou d'amis. » Il n'est pas douteux que le chapitre de cet ouvrage consacré aux classes sociales contienne une excellente ethnographie de la bourgeoisie de robe. Ce qui est étonnant, c'est son caractère d'ingénuité non critique. Halbwachs décrit des traits de comportement qui vont assez dans le sens de cette histoire vécue qu'aime à raconter mon excellent collègue Marcel Roncayolo : jeune examinateur du baccalauréat en Corse, il se fait aborder par le père d'un candidat qui le félicite de si bien examiner et lui tend discrètement sa carte de visite de juge avec ce commentaire, en aparté : «On ne sait jamais... »

29. Cf. Les Cadres sociaux, op. cit. supra n. 4, p. 332 : c'est « dans les milieux familiaux et mondains [...] que le social se crée sous les formes les plus pures, c'est là qu'il circule à travers les autres groupes. Il est naturel que les hommes qui y séjoument en soient profondément modifiés et que, quand ils se regroupent dans les cadres professionnels, ils y apportent les idées, les points de vue et tout l'ordre d'appréciations de leurs familles ou de leur monde $»$. 
les strates. La noblesse est complètement exemptée de la nécessité de travailler, c'est-à-dire de s'utiliser comme instrument au service de fins extérieures; son espace est intégralement spirituel et social. La bourgeoisie du secteur privé et du secteur public n'en est déjà plus complètement indemne, sans effort de sublimation de sa part. La matière envahit plus encore l'espace des classes moyennes, composées de petits fonctionnaires, de petits commerçants et employés, de petits industriels. Elle opère son invasion sur le monde métaphorique de l'aspect « mécanique " et anonyme que revêtent les personnes lorsqu'elles ne sont traitées que comme des usagers, mais aussi déjà, sur le mode direct, comme par exemple dans le cas de l'employé garçon-boucher qui façonne sa viande, ou du caissier qui empile et manipule... des tas de pièces d'or.

Mais la coupure décisive apparaît entre « la société ", d'une part, et les ouvriers et les paysans, d'autre part. Chez l'ouvrier, comme on l'a rapporté à maintes reprises, le contact avec la matière est exclusif : tandis que les dirigeants des entreprises et leurs collaborateurs sont en interaction sociale entre eux-mêmes et avec les ouvriers à qui ils donnent des ordres, les ouvriers sont en bout de chaîne sociale, et n'ont d'autre latitude que d'exécuter les ordres reçus en se tournant entièrement vers la matière qui les absorbe. Ainsi en est-il aussi des paysans, selon une modalité particulière qui les définit dans un "genre de vie » plutôt que dans une classe, tant ils sont isolés et disséminés sur le sol. Leur subordination à l'égard de la matière-terre se manifeste jusque dans l'établissement des constructions. Les fermes, en effet, ne sont pas d'abord des domiciles qu'on habite, ce sont des instruments de travail, disposés, non en rues comme dans les villes, ces hauts lieux de la socialité, mais dans le désordre qu'imposent les contraintes des terres à travailler. Chez les paysans et les ouvriers, la matière occupe la presque totalité de l'espace et du temps. Ce qui reste de socialité est si restreint qu'on peut le comparer à la socialité simple des primitifs.

En vertu de la spécificité et de l'autonomie du social, Halbwachs pose qu'une classe sociale ne se définit que négativement par ses conditions objectives et matérielles (son revenu, le point d'application de son activité professionnelle, etc.). Elle ne se définit positivement que par la conscience de classe, qui est pleinement du ressort de la psychologie collective ou de la sociologie. Halbwachs va même jusqu'à considérer que la bourgeoisie est capable de spiritualiser la matière, dans la mesure où l'exercice des professions bourgeoises se pénètre de socialité. Mais, lié par les présupposés aristocratiques de sa théorie, il ne peut faire entrer le travail de la bourgeoisie dans la sociologie qu'en faisant abstraction du rôle de direction de cette bourgeoisie dans la transformation démiurgique de la matière et dans le développement du capitalisme! 
Évoquant Max Weber, qu'il admire beaucoup, et Thorstein Veblen, qui l'amuse, il ne retient d'eux que l'idée selon laquelle, ce qui est socialement respecté dans une fortune, qui est le but de toute activité capitaliste, ce n'est pas "la quantité de biens matériels, quel que soit le possesseur ", mais "le mérite présumé du possesseur " ${ }^{30}$. Le capitaliste n'entre dans la sociologie de Halbwachs qu'à partir du moment où il est considéré, non pas comme quelqu'un qui intervient dans l'appropriation et dans la production des biens et des fortunes, mais seulement comme quelqu'un qui cultive, pour elles-mêmes, les vertus nécessaires à cette intervention et comme si, grand seigneur, il pouvait se permettre d'être indifférent au résultat $^{31}$ ! Non pas comme celui qui exploite un filon ou une entreprise, mais uniquement comme un faiseur d'exploits et une personne de mérite.

Mais la transposition du modèle aristocratique et la contre-offensive de l'esprit dans les professions ne sont pas l'apanage de la classe ouvrière. Si dans le cas de la bourgeoisie et aussi dans les classes moyennes, quoique de façon plus indécise, les préoccupations authentiquement sociales du monde extra-professionnel parviennent à pénétrer dans la zone des professions, ce n'est pas ce qui se passe dans le cas des ouvriers et des paysans. C'est même tout l'inverse : « une partie des habitudes de penser ou de ne pas penser, qu'entraine le contact exclusif avec la matière, reflue dans la zone de la société où vit l'ouvrier hors de l'atelier $"{ }^{32}$. Autrement dit, dans les " classes hautes " de la société, ce n'est pas la fortune (indice économique et matériel) qui indique l'appartenance de classe sociale, mais "la dépense ", et même la dépense ostensible ${ }^{33}$, qui est la fortune reconnue, la fortune en représentation psycho-sociale. Au-delà de la " coupure " où campe la classe ouvrière, une inversion se produit :

« il semble que ce ne soit pas la même cause qui du haut en bas de la série détermine quelles fortunes se grouperont pour former une classe, et qu'on tienne compte, pour les basses classes, surtout du travail salarié, pour les hautes, de la dépense ${ }^{34}$.

30. Ibid., p. 346 sq.

31. Cf. ibid., p. 340 sq. : la fortune matérielle n'est pas un indice social, sinon, "les hommes, confondus avec les biens, seraient confondus avec les choses ". Illustration : il faut supposer qu' « un riche est riche parce qu'il est qualifié pour l'être ", et non pas parce que sa condition de riche est déterminée par la possession, matérielle et contingente, d'une fortune. Cette métamorphose prend du temps : " tandis qu'une fortune, économiquement, est tout de suite ce qu'elle est, tandis qu'elle peut se construire ou se détruire en quelques jours [...] ou en quelques instants [...], socialement elle ne compte, et on n'en tient compte, dans les milieux du monde, qu'au bout d'un certain temps $»$. Le nouveau riche ne fait partie ni de la sociologie ni de la société...

32. Ibid., p. 333.

33. Cf. « Remarques sur la position du problème sociologique des classes », art. cit. supra n. 4.

34. Ibid., p. 45. 
Faut-il voir, dans le point d'inflexion de cette hélice théorique, la manifestation d'une inconséquence logique ? Il semblerait, en effet, que Halbwachs applique correctement, dans le cas des classes hautes, le principe selon lequel le social échappe à la causalité extérieure de l'économique et du matériel et qu'il ne le fasse plus dans le cas de la classe ouvrière.

Paradoxalement, la démarche de Halbwachs n'est pas inconséquente, dans la mesure où la causalité matérielle du social qu'elle met au jour dans le cas des ouvriers, et qui détermine leur conscience de classe, est sans conséquence pour l'intégrité de l'idéalisme sociologique, puisqu'elle n'est jamais que négative. C'est du néant, et le contenu social de la conscience de classe ouvrière se réduit presque à rien: Halbwachs le nomme solidarité ( une vague solidarité ») pour indiquer qu'il ne s'agit que d'une socialité fruste. La matière occupe presque toute la place dans la vie des ouvriers, qui finissent par ressembler, dans leur aspect physique, aux instruments mécaniques qu'ils manipulent. Chez des êtres pénétrés de matière, ne peut se développer que «le plus bas degré de la conscience sociale ", et l'ensemble de leurs rapports " imite les relations mécaniques entre les choses". Dans ces « couches sociales (à peine sociales) inférieures ", " le besoin sexuel n'est plus que physique » et, vu la faible part que lui réservent les budgets ouvriers, le logement n'est plus un " abri social ", mais " il redevient un lieu de vie animale, et comme une annexe de l'usine où l'homme se trouve hors de la société ». D'où la négligence que les ouvriers affichent pour la vie du " home ", la vie de famille et le confort intérieur; et leur prédilection pour la déambulation dans les rues, qui représentent « très littéralement le dehors, c'est-à-dire le monde extérieur » où l'individu n'est « qu'un morceau de matière en mouvement ${ }^{35}$.

En inscrivant la combinatoire de la profession et du revenu dans le dualisme de l'aristocratie (considérée comme l'essence du social) et de la matière (considérée comme le pôle absolu de la désocialisation), Halbwachs ne fait sans doute qu'exprimer ses propres préjugés de petit bourgeois fasciné par le mode de vie aristocratique tel qu'il se prolongeait dans la bourgeoisie de robe de son temps ${ }^{36}$.

35. Toutes ces citations sont extraites du Livre III de La Classe ouvrière et les niveaux de vie, op. cit. supra n. 4, passim.

36. Le magistrat de justice symbolise la bourgeoisie de facon récurrente sous la plume d'Halbwachs; of. Les Cadres sociaux de la mémoire, Les Caractéristiques des classes moyennes, etc. Tout ce qu'il décrit des mours et conduites de cette bourgeoisie est à prendre comme la restitution du vécu interne de cette bourgeoisie, et comme le point de vue subjectif de cette bourgeoisie sur ses rapports fantasmatiques avec l'ancienne noblesse, d'une part, et avec la classe ouvrière, d'autre part. 
Une limitation aussi arbitraire de la définition des dimensions de la socialité ne permettait malheureusement pas à la sociologie industrielle ultérieure de prendre appui sur les travaux de Halbwachs. La surimposition du dualisme aristocratique du noble et de l'ignoble au dualisme idéaliste de la matière et de l'esprit est une pièce rapportée tout à fait dissociable de la théorie durkheimienne de l'autonomie spécifique du champ social et de la définition du fait social comme représentation psychique, même s'il est patent qu'une pièce de ce calibre est loin de jurer avec l'idéalisme durkheimien. À cet égard, Halbwachs n'est pas régressif par rapport à Marx mais à Durkheim ${ }^{37}$ !

Nous n'avons pas de réponse à la question que pose cet arrimage énigmatique du système sociologico-philosophique de Halbwachs au pôle aristocratique. Entre la bourgeoisie, qui reprend à son compte les valeurs de la noblesse, et l'ensemble des paysans et des ouvriers qui pourraient bien descendre de la nation autochtone vaincue par de nobles envahisseurs ${ }^{38}$, Halbwachs insère les classes moyennes, dont il énumère les diverses composantes. Curieusement, les professeurs ne figurent nulle part, ni dans la bourgeoisie, ni dans les classes moyennes. Le classeur n'est pas dans son classement. Il ne se voit pas, ou ne veut pas se voir. Le professeur d'université qu'il était, fils de professeur de l'enseignement secondaire, n'a-t-il pas osé s'auto-recruter dans la bourgeoisie aristocratisée de son tableau, ou a-t-il été gêné de s'afficher dans les classes moyennes?

Certainement, en tout cas, il éprouve de la fascination pour la socialité mondaine de la bourgeoisie et, s'il parle tant de la classe ouvrière, c'est qu'il a eu pour elle des sympathies politiques, tout au moins au début de sa carrière, comme l'attestent les articles qu'il a publiés dans la Revue socialiste avant $1914^{39}$.

37. Pour libérer les potentialités que contenait la pensée d'Halbwachs, il suffirait d'admettre que rien de ce qui est humain n'est exclu, en fait comme en droit, de la nature sociale, et que le travail et la transformation de la matière ont une signification sociale dès lors qu'ils sont ceuvre humaine.

38. L'hypothèse est suggéree dans l'Introduction du Cours de la Sorbonne sur Les Classes sociales, op. cit. supra n. 4.

39. Dans La Mémoire collective, op. cit. supra n. 6, p. 49, Halbwachs évoque des souvenirs de conversations d'enfance qu'il a eues avec une vieille bonne, qui s'exprimait à la manière des domestiques, "comme de grands enfants ". C'est par elle qu'il a entendu parler de la Guerre de 1870, de la Commune, du Second Empire, de la République : « pleine de superstitions et de partis pris, [elle] acceptait sans discussion le tableau de ces événements et de ces régimes qui avait été peint par l'imagination populaire. Par elle me parvenait la rumeur confuse qui est comme le remous de l'histoire qui se propage dans les milieux de paysans, d'ouvriers, de petites gens. Mes parents, quand ils l'entendaient, pouvaient hausser les épaules. [...] Si je reconnais à présent à quel point ces récits étaient inexacts, je ne puis faire que je ne me sois penché alors sur ce courant trouble... " Et sur les ouvriers, par la suite? 


\section{IV. - LA SOCIETtE ET SES DEUX MATIÈRES}

En fait, jusqu'à présent, nous n'avons repéré dans la pensée de Halbwachs qu'une grave lacune : à trop insister sur la ségrégation de la classe ouvrière par rapport à la société civilisée, elle s'aveugle sur tout un univers de dimensions de la socialité. Mais il y a plus : cette matière, que Halbwachs rejette avec la classe ouvrière aux portes de la société, revient, si l'on peut dire, par les fenêtres; et sa présence obsédante entraîne la pensée de Halbwachs dans de curieuses contorsions.

Évoquons d'abord une rencontre quasi surréaliste. On a rappelé la contribution importante que Halbwachs a apportée à l'élaboration de la morphologie sociale. Bien plus, l'étude de la «forme matérielle des sociétés » n'est pas un chapitre secondaire de la sociologie :

« L'auteur des Règles de la méthode sociologique, qui recommandait d'étudier les réalités sociales "comme des choses", devait attribuer une impor-

Peut-être la brutalité de la coupure, que Halbwachs met un soin perpétuel à maintenir entre la classe ouvrière et la part vraiment sociale de la société, exprime-t-elle en partie une réaction de défense de type " petit blanc " d'une catégorie en situation de descente sociale et acharnée à préserver sa différence par rapport à la strate sociale inférieure ? Quoi qu'il en soit, l'idée que la classe ouvrière est une horde non civilisée, qui campe aux lisières de la cité, a hanté le XIX 'siècle et ne s'est peut-être vraiment affaiblie qu'après le Front populaire et la Seconde Guerre mondiale (en poursuivant une seconde vie dans le registre de l'immigration). Pour tout dire, ce qui nous heurte, chez un sociologue de la stature de Halbwachs, c'est qu'il ait endossé ce schème dualiste de la bourgeoisie civilisée et de la classe ouvrière non civilisée, sans esprit critique : sa fascination est égale des deux côtés, négative ici, positive là. Si Halbwachs avait pu connaître de moins loin la classe ouvrière, il aurait constaté que même encore dans les années 30-40, le couple civilisé/non civilisé (qu'il utilise comme catégorie à la fois spontanée et construite) fonctionnait aussi d'une couche à l'autre, voire, dans la couche inférieure, d'une sous-couche à l'autre : des familles de mancuvres français pouvaient toujours se sentir confortées dans leur indentité de civilisés, par différence avec les familles voisines d'immigrés. Même les plus démunis reprennent à leur compte l'opposition du civilisé et du non civilisé. Témoin cette réflexion que, plus près de nous, dans On est tous dans le brouillard. Ethnologie des banlieues, Paris, Galilée, 1979 et 1985, p. 97, Colette PETonnet recueille auprès d'une famille de manceuvres en cité de transit : elle pose la question « utilisez-vous des serviettes de table? »; on lui répond : « Non. Chez nous, on mange proprement. On n'a pas besoin de serviettes. "

Le débat, pour nous aujourd'hui, présuppose que soit reconnue l'intégration de la classe ouvrière à la culture et à la socialité commune. Ce qui n'empêche pas de s'interroger sur l'existence d'une spécificité de la culture ouvrière ou sur la capacité de la culture dominante à empêcher tout développement positif d'une subculture spécifique. Voir sur ce point : Claude Grignon et Jean-Claude PASSERon, Sociologie de la culture et sociologie des cultures populaires, Cahiers du C.E.R.C.O.M., Paris, École des hautes études en sciences sociales/Vieille Charité (2, rue de La Charité, 13002 Marseille), 1982, et M. VERrET, La Culture ouvrière, 34230 Saint-Sébastien-sur-Loire, Éd. A.C.L., 1988. 
tance particulière à ce qui, dans les sociétés, emprunte davantage les caractères des choses physiques : étendue, nombre, densité, mouvement, aspect quantitatif, tout ce qui peut être mesuré et compté $"{ }^{40}$.

Soit. Mais quelle signification attribuer, dans ce contexte, à une formule comme celle-ci, qu'on rencontre dans l'essai sur les caractéristiques des classes moyennes ${ }^{41}$ : "l'essentiel de leur activité est de traiter [les autres hommes] comme des choses " ? De fins sociologues durkheimiens, les employés de bureau? Halbwachs ne s'est pas expliqué sur cette coïncidence. Mais, en revanche, il éprouve le besoin de répondre longuement à une objection qu'il se formule à lui-même, lorsqu'il constate l'existence d'un parallélisme entre la posture du physicien qui travaille à connaître la matière, et celle de l'ouvrier manuel, qui travaille à la transformer : « $\grave{A}$ ces moments, le savant est bien tourné vers la matière, exactement comme l'ouvrier manuel $"{ }^{42}$.

Si ce parallélisme est fondé, comment exclure l'ouvrier de la société et y conserver le physicien ? C'est bien une matière étrangère à l'esprit que le physicien connaît, dit Halbwachs; mais dans la théorie de la matière se trouvent résumées "l'expérience et les tentatives d'explication d'une société qui se continue dans celle d'aujourd'hui ». Mais alors, pourquoi n'y aurait-il pas l'équivalent de cette mémoire collective, d'ordre spirituel, dans le « savoir empirique et les réflexions sur le métier auxquels le travailleur peut s'élever " ? Halbwachs ne peut répondre négativement qu'en endossant à découvert le préjugé ethnocentrique de la bourgeoisie de son temps : il n'y a pas d'équivalence, dit-il en substance, parce que la société refuse d'en reconnaître une et parce qu'elle a poussé " trop loin » la distinction entre activités manuelles et intellectuelles pour qu'une telle équivalence soit acceptable.

Le système de pensée de Halbwachs pouvait difficilement concilier les deux exigences polaires de la sociologie durkheimienne, qui commandent d'identifier le social au psychique (collectif), mais de le traiter méthodologiquement comme s'il épousait la nature des réalités physiques. La cause de cette faiblesse réside, croyons-nous, dans l'interprétation extrémiste qu'il se donne des deux termes polaires. Halbwachs conçoit l'esprit collectif comme un esprit qui ne travaille pas et qui ne se développe idéale-

40. Avant-propos de Morphologie sociale, 1938, op. cit. supra n. 1. Il y a confusion dans le propos de Halbwachs : étudier tous les faits sociaux sous l'aspect par lequel ils ressemblent aux choses, ce n'est pas étudier les seuls faits sociaux qui ressemblent aux choses.

41. Paru en 1939 in Inventaires III, op. cit. supra n. 24, republié dans Classes sociales et morphologie, op. cit. supra n. 1, p. 95-111, en part. p. 107.

42. Dans « Matière et société », art. cit. supra n. 10, p. 86 ; de même que les citations de l'alinéa suivant. 
ment que dans le loisir perpétuel ${ }^{43}$. L'écart est le plus grand possible entre une conception de la spécificité des faits sociaux qui sont identifiés à l'interconnaissance des personnes singulières liées au sein du tissu vivant des générations, et la règle de méthode qui commande de saisir les faits sociaux sous leur aspect de choses, résistantes et mesurables.

La matière joue aussi un rôle bénéfique, car elle est à la société ce que le corps est à l'âme, son principe de stabilité et d'équilibre ${ }^{44}$. Il n'empêche que ce contact avec les choses doit être médiatisé :

"C'est peut-être une nécessité qui s'impose au corps social de détacher ainsi, au moins en partie, un ensemble [...] de ses membres, ceux qui doivent être mis en contact avec les choses. La société prend place au milieu des choses, mais justement elle veut éviter leur contact, et alors, la seule façon qu'elle ait d'y parvenir, c'est de se dédoubler, d'obliger une partie d'ellemême à se soumettre à ce contact pénible avec les choses $[\ldots]$ ". ${ }^{45}$.

Ce tampon protecteur n'existe pas dans le rapport que les sociétés entretiennent avec les facteurs physiques et biologiques réfractés dans le comportement démographique. Les sociétés prennent, de l'espace et des besoins organiques, une conscience authentiquement collective, même si elle est parfois obscure, et transmettent à leurs membres les orientations déposées dans cette conscience et qui les font agir socialement, c'est-àdire émigrer ou non, procréer davantage ou restreindre les naissances, etc.

Ce qui étonne le plus dans ce système, ce sont les limitations qu'il s'inflige. Qu'au lieu de concevoir la matière comme invasion barbare à contenir, on la conçoive comme objet de conquête sociale, et le système, au lieu de se refermer stérilement ou de rester écartelé, s'ouvre à tous les développements possibles! Encore faudrait-il se représenter le «foyer central " de la société, non comme l'âtre du culte des ancêtres, mais

43. Il ne brûle que pour lui-même, du feu de la mémoire collective; il n'a rien de commun avec le feu prométhéen du travail de la transformation de la matière. Une image allégorique, exposée dans l'Introduction de La Classe auvrière et les niveaux de vie, op. cit. supra n. 4 , p. Iv-v, contient, ramassée, toute la pensée de Halbwachs sur la société (moins l'ajout aristocratique). La société tout entière, selon Halbwachs, peut être regardée comme si elle était sous l'emprise d'un mouvement de double sens : " ou bien on dira que la société dans son ensemble tend à se dépasser, que la vie sociale, d'abord diffuse, éparpillée, soumise à l'action de beaucoup de forces de dispersion, petit à petit se concentre, se ramasse comme autour d'un foyer qu'elle a elle-mème allumé et qu'elle alimente; ou bien on dira que la société fait un effort et un effort pénible et constant, pour se distendre, comme s'il lui fallait écarter d'elle beaucoup de forces d'oppression qui l'enserrent et l'étouffent, et que les parties d'elle-même les plus voisines de la périphérie se trouvent de plus en plus éloignées du foyer central, de plus en plus en contact avec le " dehors", qu'elles perdent en même temps de leur souplesse et de leur élasticité, qu'elles se durcissent et qu'elles se figent. "

44. Cf. Morphologie sociale, op. cit. supra n. 1, passim.

45. Cf. Les Classes sociales, op. cit. supra n. 4, p. 60. 
comme un haut fourneau (si l'on tient à l'image du feu, bien entendu) et la classe ouvrière autrement que comme un cordon sanitaire entre la spiritualité sociale et la matière. Halbwachs n'a pas reconnu que la transformation collective de la nature était une œuvre sociale, c'est-à-dire une œuvre qui provoquait en retour la transformation de la société dans toutes ses parties.

Michel Aмıт, Centre d'étude des mouvements sociaux, École des hautes études en sciences sociales. 